This item was submitted to Loughborough's Research Repository by the author.

Items in Figshare are protected by copyright, with all rights reserved, unless otherwise indicated.

\title{
Characterising variability and regional correlations of microstructure and mechanical competence of human tibial trabecular bone: An in-vivo HR- pQCT study
}

\section{PLEASE CITE THE PUBLISHED VERSION}

https://doi.org/10.1016/j.bone.2019.01.013

\section{PUBLISHER}

(C) Elsevier BV

\section{VERSION}

AM (Accepted Manuscript)

\section{PUBLISHER STATEMENT}

This paper was accepted for publication in the journal Bone and the definitive published version is available at https://doi.org/10.1016/j.bone.2019.01.013.

\section{LICENCE}

CC BY-NC-ND 4.0

\section{REPOSITORY RECORD}

Du, Juan, Katherine Brooke-Wavell, Margaret A. Paggiosi, C. Hartley, Jennifer S. Walsh, Vadim Silberschmidt, and Simin Li. 2019. "Characterising Variability and Regional Correlations of Microstructure and Mechanical Competence of Human Tibial Trabecular Bone: An In-vivo Hr-pqct Study”. Loughborough University. https://hdl.handle.net/2134/36668. 


\section{Characterising variability and regional correlations of}

\section{microstructure and mechanical competence of}

\section{human tibial trabecular bone: an in-vivo HR-pQCT}

\section{study}

Juan $\mathrm{Du}^{1}$, Katherine Brooke-Wavell ${ }^{2}$, Margaret A Paggiosi ${ }^{3}$, Chris Hartley², Jennifer S Walsh $^{3}$, Vadim V Silberschmidt ${ }^{1}$, Simin $\mathrm{Li}^{1}{ }^{1}, *$

${ }^{1}$ Wolfson School of Mechanical, Electrical and Manufacturing Engineering, Loughborough University, Leicestershire, UK

${ }^{2}$ School of Sport, Exercise and Health Science, Loughborough University, Leicestershire, UK

${ }^{3}$ Mellanby Centre for Bone Research, University of Sheffield, Sheffield, UK

* Corresponding author. Email address: S.Li@lboro.ac.uk

\section{Highlights}

- A two-phase FEA model with individual trabecular representation was created to estimate regional trabecular stiffness.

- Significant regional variation of trabecular stiffness of human distal tibia was quantified.

- All regional parameters correlated significantly with regional stiffness, with regional BV/TV showing highest correlation.

- Medial \& posterior had higher stiffness and trabecular indices in BV/TV, Tb.Th and Tb.BS, than anterior \& central regions.

- Medial and posterior regions had more plate-like trabecular structures than rodlike ones. 


\section{Abstract}

Objective: Quantifying spatial distribution of trabecular bone mechanical competence and microstructure is important for early diagnosis of skeletal disorders and potential risk of fracture. The objective of this study was to determine a spatial distribution of trabecular mechanical and morphological properties in human distal tibia and examine the contribution of regional variability of trabecular microarchitecture to mechanical competence.

Methods: A total of 340 representative volume elements at five anatomic regions of trabecular bone - anterior, posterior, lateral, medial and centre - from ten white European-origin postmenopausal women were studied. Region-specific trabecular parameters such as trabecular volume fraction, trabecular thickness, trabecular number, trabecular surface area, trabecular separation, plate-like structure fraction and finite element analysis of trabecular stiffness were determined based on in-vivo high resolution peripheral quantitative computed tomographic (HR-pQCT) images of distal tibiae from ten postmenopausal women. Mean values were compared using analysis of variance. The correlations between morphological parameters and stiffness were calculated.

Results: Significant regional variation in trabecular microarchitecture of the human distal tibia was observed $(p<0.05)$, with up to $106 \%$ differences between lowest (central and anterior) and highest (medial and posterior) regions. Higher proportion of plate-like trabecular morphology (63\% and $53 \%)$ was found in medial and posterior regions in the distal tibia. Stiffness estimated from finite element models also differed significantly $(p<0.05)$, with stiffness being 4.5 times higher in the highest (medial) than 
lowest (central) regions. The bone volume fraction was the strongest correlate of stiffness in all regions.

Conclusion: A novel finding of this study is the fact that significant regional variation of stiffness derived from two-phased FEA model with individual trabecula representation correlated highly to regional morphology obtained from in-vivo HR-pQCT images at the distal tibia. The correlations between regional morphological parameters and mechanical competence of trabecular bone were consistent at all regions studied, with regional BV/TV showing the highest correlation. The method developed for regional analysis of trabecular mechanical competence may offer a better insight into the relationship between mechanical behaviour and microstructure of bone. The findings provide evidence needed to further justify a larger-cohort feasibility study for early detection of bone degenerative diseases: examining regional variations in mechanical competence and trabecular specifications may allow better understanding of fracture risks in addition to others contributing factors.

Key words: HR-pQCT; Regional variation; Mechanical competence; Finite-element analysis; Multiple variable regression; Trabecular microstructure 


\section{Introduction}

Osteoporosis is a common metabolic bone disease, causing bone fragility and increasing risk of bone fracture [1]. Research on early identification and diagnosis of osteoporosis with patients at high risk for fracture is needed to reduce clinical consequences associated with osteoporotic fractures. The main characteristics of osteoporotic bone are reduction in bone density and mass as well as deterioration of both cortical and trabecular microstructures [2]. These changes are often reported as regional [3][4] and non-uniform [5]. Currently, dual X-ray absorptiometry (DXA) scan, is the only method recommended by the World Health Organization (WHO) to assess fracture risk and osteoporosis [6][7]. However, the standard way to use DXA to estimate the risk of fracture is based on a single value of areal bone mineral density; information about spatial variation of bone microstructure and mechanical competence is not provided.

Localised variations in loading and anatomy are the primary cause of the heterogeneous distribution of bone mass and its microstructure. These variations in morphology could further impact the local mechanical properties such as stiffness and strength, resulting in varying propensity to fracture at different anatomical locations. A number of studies on the anatomic variance of cortical bone's properties have been conducted both in-vivo and in-vitro [8][5][9][10][11]. However, in a recent study, it was suggested that trabecular bone rather than cortical bone was most strongly associated with fracture risk [12]. It has been reported that spatial distribution of microstructural elements in trabecular bone is compromised in fracture patients [13][14][15]. Thus, understanding spatial variation of trabecular microstructure may provide potential improvements in fracture prediction [16] . 
The development of high-resolution peripheral quantitative computed tomography (HR-pQCT) enables the assessment of 3D microstructure of trabecular bone in-vivo. This imaging modality provides 3D data sets superseding the resolution of traditional clinical QCT (86 $\mu \mathrm{m}$ versus $250-500 \mu \mathrm{m})$ [17]; it permits separation of corticaltrabecular regions, and further quantification of density and structural parameters at peripheral sites[18]. It opens up a wide range of studies, including but not limited to aging [18][19][20][16][21] and the effects of various of pathologies on trabecular bone[22][23][24]. However, most HR-pQCT studies have evaluated the microstructure of trabecular bone at a global level, assuming a homogenised structure for the entire scan area. In fact, trabecular microstructure varies significantly not only across the sagittal plane [25], but also in the transverse plane [26][27]. Sode and Burghardt et al. [27][28] studied differences of regional variations in trabecular structure due to gender, age and alendronate effects using HR-pQCT and found that, in all gender and age groups, trabecular bone in the medial and posterior region of distal tibia exhibits higher values of BV/TV, Tb.N, Tb.Th and low Tb.Sp compared to global averages. Although this study indicated that the trabeculae are heterogeneously distributed across the region, the regional variation of mechanical competence has not been described, and the contribution of regional microstructure variations to regional variations in mechanical competence is still unknown.

By segmenting the 3D in-vivo HR-pQCT scans of the human distal tibia anatomically, regional quantification provides an insight of structural-mechanical relationship and material heterogeneity of trabecular bone. We hypotheses that heterogeneity exists among anatomical regions, with stiffness estimated from FE analysis significantly higher in the medial and posterior regions than other regions. Compared with the global assessment, regional stiffness exhibited better correlation with regional 
microarchitecture. The objective of this study was to quantify the regional variation of trabecular morphology mechanical properties and to explore the potential role of trabecular microarchitecture on mechanical competence in five distinct anatomical regions.

\section{Materials and methods}

\subsection{Participants and HR-pQCT imaging}

Participants were ten healthy white European-origin women, with a mean age (and standard deviation) of $63.3 \pm 3.6$ years, height of $1.60 \pm 0.10 \mathrm{~m}$ and weight of $67.3 \pm$

$5.7 \mathrm{~kg}$, with no diagnosed or symptomatic fractures, osteoporosis or any musculoskeletal injury, recruited from the local community. All participants gave their written informed consent, and the study was approved by the Ethics Approvals (Human Participants) Sub-Committee at Loughborough University, UK and the National Research Ethics Service.

The distal tibia of both legs of the participants was scanned using HR-pQCT (XtremeCT, Scanco Medical) with a standard in-vivo scanning protocol (60 kVp, 1000 mA, 100-msintegration time) at the NIHR Clinical Research Facility, Sheffield Teaching Hospitals NHS Foundation Trust, Sheffield. The region of interest was identified from a 2D scout-view, $22.5 \mathrm{~mm}$ proximal to the distal tibia endplate (Fig. 1a). Each scan contained 110 equally distributed slides along a $9.02 \mathrm{~mm}$ axial length, with an isotropic voxel size of $82 \mu \mathrm{m}$. Prior to image acquisition, the tibia was immobilized in a carbon fibre cast and fixed within the gantry of the scanner to ensure the correct position upon entry. A post-scan quality check was performed based on motioninduced image artefacts: a quality scale was defined from grade 0 (no motion) to grade 
4 (significant blurring of the periosteal surface, discontinuities in the cortical shell, or streaking in the soft tissue) [29]. In this study, images defined as grade 4 were rejected and the scan was repeated once to ensure only good quality images were used during the analysis procedures.
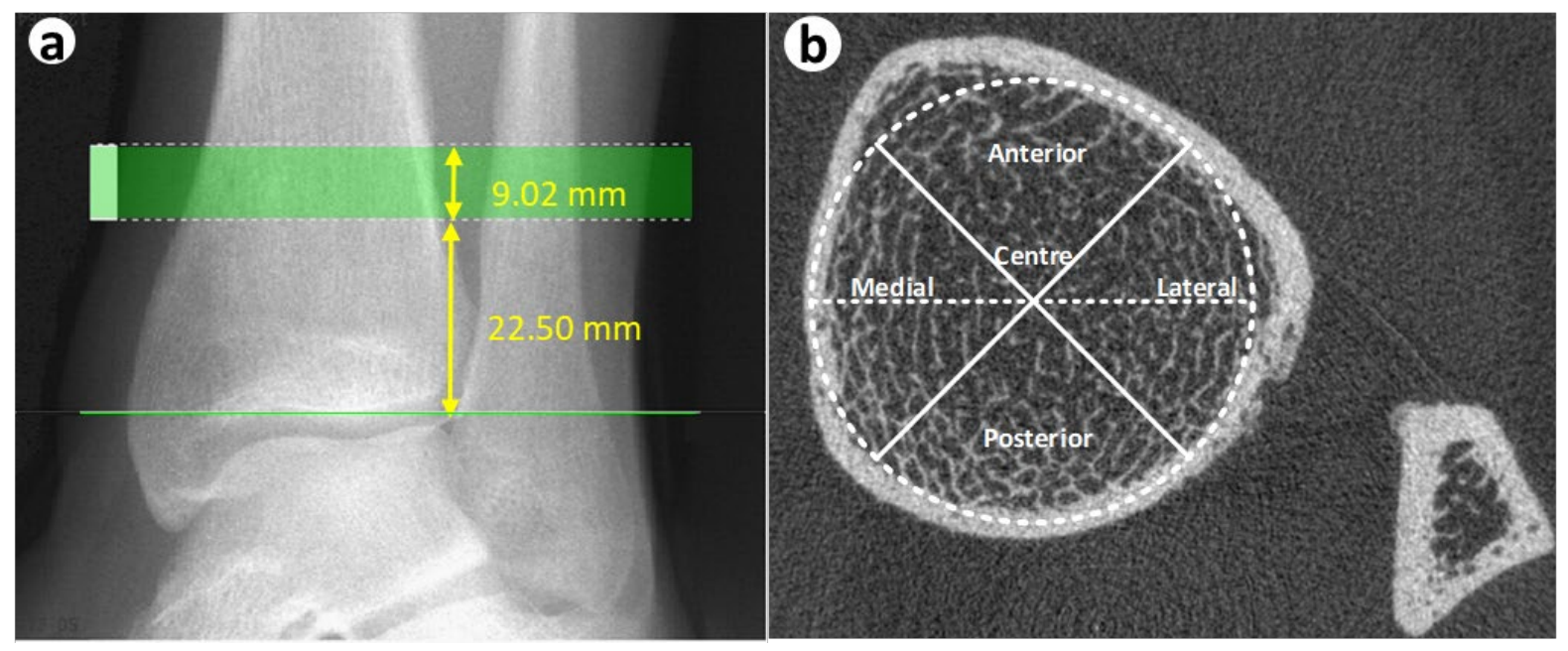

Figure 1: (a) An anterior-posterior scout-view with the HR-pQCT scanner of a distal tibia with a marked tibia endplate (green solid line) and the region of interest (between two green dashed lines); (b) anatomic regions of interest for distal tibia obtained from the median slide of a typical HR-pQCT scan. A circle (thicker dashed line) was used to fit the outer surface of tibia to find the geometric central point; a horizontal line (thin dash line) was then drawn through the centre of the circle together with two lines at $45^{\circ}$ and $-45^{\circ}$ to the horizontal one; five anatomical regions (anterior, posterior, lateral, medial and centre) were denoted with these two lines.

\subsection{Analysis of local and global microstructure}

Region-specific morphological features of the studied trabecular bone were analysed in this study at five anatomical regions: anterior $(A)$, posterior $(P)$, lateral $(L)$, medial (M) and centre (C) (Fig. 1b). A cubic representative volume element (RVE) with a sidelength of $4 \mathrm{~mm}$ was used to ensure that cross-comparison between participants with bones of different shapes and sizes was possible and it contained sufficient microstructure. A total of three hundred and forty RVEs, eighty RVEs for each anatomical region $(n=80)$, and twenty from the centre of each leg from the ten participants, were analysed in this study (Fig. 2). The selected RVEs were evenly distributed within each participant, either along the centre line of each region or spread 
at equal distances away from the inner surface of the cortical bone. A similar pattern of RVE distribution was implemented across all participants thanks to anatomical similarity of distal tibiae and all the cubes were carefully visually checked to ensure that they contain no cortical bone region.

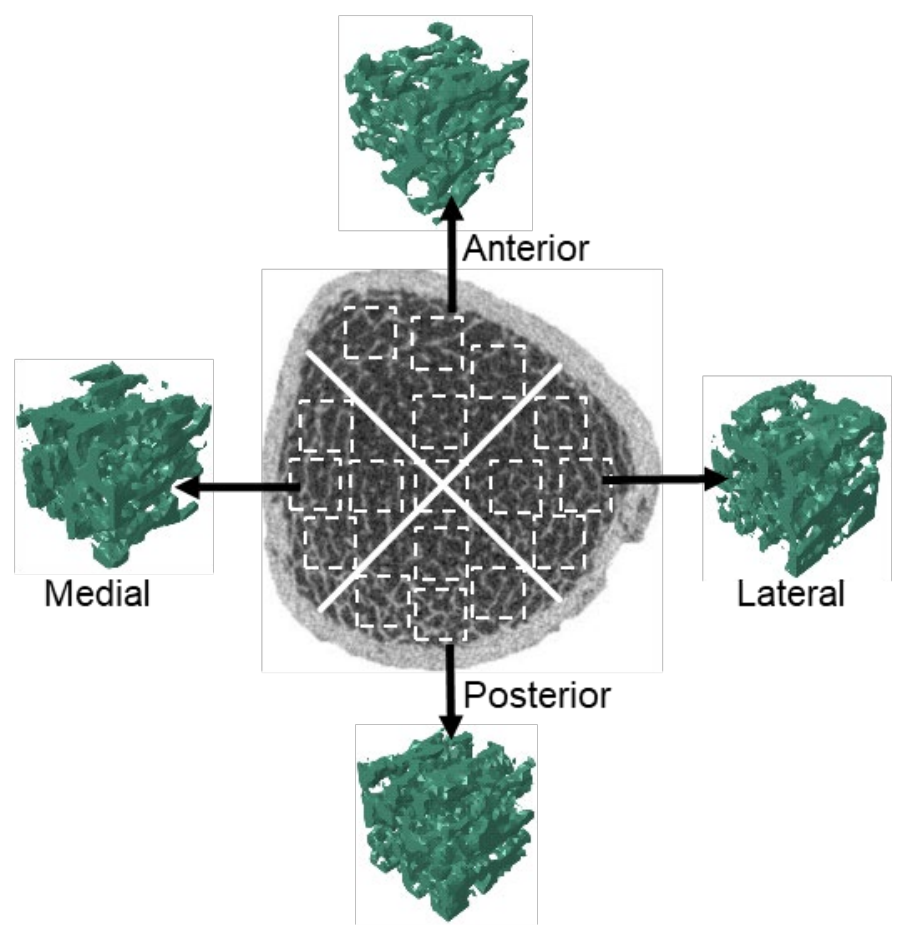

Figure 2: Diagram of the location of each RVE (four cubes with a side length of $4 \mathrm{~mm}$ were extracted from each anatomic region and one from the centre. Around $60 \%$ of this cross-sectional area was covered by RVEs.

Image segmentation and reconstruction were performed using Materialise Mimics Innovation Suite 19.0 (Materialise, Leuven, Belgium), and trabecular compartments were extracted based on a fixed threshold corresponding to $300 \mathrm{mg} \mathrm{HA} / \mathrm{cm}^{3}$ used in global measurements [30]. Local three-dimensional (3D) trabecular microstructures were analysed using BoneJ, an open-source plugin in Image $J$ [31]. Standard trabecular bone microstructures parameters were analysed directly for every RVE, as listed in Table 1: trabecular volume fraction (BV/TV), trabecular thickness (Tb.Th) and trabecular space (Tb.Sp), number of trabeculae per millimetre (Tb.N), trabecular bone surface area (Tb.BS) and plate-like structure fraction ( $\mathrm{PTb} . \mathrm{N} / \mathrm{TTb} . \mathrm{N})$. It should be noted 
that the plate-like structure fraction (PTb.N/TTb.N), defined as the number of plate-like structures divided by the total number segmented structures, was introduced to quantitatively evaluate the variation of topological characteristic of trabecular structure among anatomical regions. A plate-like trabecula is determined when the ellipsoid factor $(E F)$ calculated in BoneJ for the given space falls between -1 and 0 , i.e. producing a flattened (oblate) ellipsoid. Detail of calculating the EF is documented in previous work [32], hence not mentioned here.

All global parameters (i.e. mean value for the entire scanned area) were evaluated using the HR-pQCT manufacturer's standard analysis protocol [18]. Briefly, a semiautomatic threshold-based algorithm was used to separate cortical from the trabecular bone compartments. Trabecular BMD (BMD trab) and BV/TVd defined as $B M D_{\text {trab }}$ divided by the assumed density of fully mineralized bone (1200 mg HA/cm${ }^{3}$ ) were obtained. It should be noted that Tb.N was the only global parameter measured directly from images, the others were dependent parameters derived (hence, superscript "d") from Tb.N and BV/TV d using a plate-model assumption, as described in Table. 1.

Table 1: Main parameters of trabecular microstructure and respective measurement methods

\begin{tabular}{|c|c|c|}
\hline $\begin{array}{l}\text { Indices } \\
\text { (units) }\end{array}$ & Definition & Measurement method \\
\hline \multicolumn{3}{|c|}{ Directly measured parameters } \\
\hline $\mathrm{BV} / \mathrm{TV}$ & $\begin{array}{l}\text { Ratio of trabecular bone } \\
\text { volume to total tissue volume }\end{array}$ & $\begin{array}{l}\text { The number of foreground (bone) voxels divided by the } \\
\text { total number of voxels in the image }\end{array}$ \\
\hline Tb.N (1/mm) & $\begin{array}{l}\text { Trabecular number: mean } \\
\text { number of trabeculae }\end{array}$ & $\begin{array}{l}\text { Inverse of the diameter of the sphere that fits the ridges } \\
\text { between trabeculae [33] }\end{array}$ \\
\hline $\begin{array}{l}\text { Tb.Th } \\
(\mathrm{mm})\end{array}$ & Trabecular thickness & $\begin{array}{l}\text { The diameter of the largest sphere that fits within a } \\
\text { trabecula [33] [34] }\end{array}$ \\
\hline $\begin{array}{l}\text { Tb.BS } \\
\left(\mathrm{mm}^{2}\right)\end{array}$ & Trabecular bone surface area & $\begin{array}{l}\text { The sum of the areas of triangles making up a surface } \\
\text { mesh [35] }\end{array}$ \\
\hline
\end{tabular}


Tb.Sp Trabecular separation: Mean ( $\mathrm{mm}$ space between trabeculae

Tb.Ar Mean area occupied by

$\left(\mathrm{mm}^{2}\right)$ trabecular bone

Ratio of plate-like structures to PTb.N/TTb.N total number of segmented structures
The diameter of the largest sphere that fits within the space between trabeculae [33] [34]

Compartments are calculated on a slice-by-slice basis and averaged over all slices [28]

The number of segmented structures with EF between 0 and -1 divided by the total number of structures [32]

\section{Derived parameters}

BV/TVd Derived ratio of trabecular

volume over total volume

Tb. Th ${ }^{d} \quad$ Derived trabecular thickness

$(\mathrm{mm})$

Tb.Spd $(\mathrm{mm})$

$$
\text { using plate-model assumption }
$$

Derived trabecular separation using plate-model assumption

$$
\mathrm{BV} / \mathrm{TV}^{\mathrm{d}}=\frac{\mathrm{BMD}}{1200 \mathrm{mgHA} / \mathrm{cm}^{3}}[36]
$$

$$
\operatorname{Tb} \cdot \mathrm{Th}^{\mathrm{d}}=\frac{{\mathrm{BV} / \mathrm{TV}^{\mathrm{d}}}^{\mathrm{d}}}{\mathrm{Tb} . \mathrm{N}}[37]
$$

$$
\mathrm{Tb} . \mathrm{Sp}^{\mathrm{d}}=\frac{1-\mathrm{BV} / \mathrm{TV}^{\mathrm{d}}}{\mathrm{Tb} . \mathrm{N}}[37]
$$

'd' indicates a parameter derived indirectly from other known parameters. Parameters highlighted with grey represent the HR-pQCT standard-analyse protocols measurements; other parameters are direct measurements with Image $\mathrm{J}$.

\subsection{Finite-element analysis of trabecular bone stiffness}

Finite-element models of trabecular microstructure based on 340 RVEs taken from five anatomic regions of the distal tibia were generated to analyse their mechanical competence in-silico. A two-phase material model including trabecular bone and marrow was considered. Both bone and marrow were modelled as isotropic, linearelastic material with Young's modulus of $15 \mathrm{GPa}$ and $3 \mathrm{MPa}$, respectively, and

Poisson's ratio of 0.3 and 0.17 , respectively [38] [39]. Approximately one million voxelbased tetrahedral elements were used to create the finite element mesh of the trabecular microstructure using 3-Matic 11.0 (Materialize, Leuven, Belgium). A global seeding of $70 \mu \mathrm{m}$ was used to ensure that elements remained within one voxel size of $82 \mu \mathrm{m}$ and that a systematic error in apparent stiffness did not reduce the ability of the finite-element model to accurately predict bone strength and correlated well with the experimental results [40][41]. A $1 \%$ uniaxial compressive strain was applied 
perpendicularly to the proximal surface of the trabecular microstructure to determine the axial stiffness of each RVEs (Fig. 3). All finite element models were simulated using Abaqus 6.14 software (Dassault Systems Simulia Crop, Providence, RI, USA) on a desktop workstation (HP Z440).

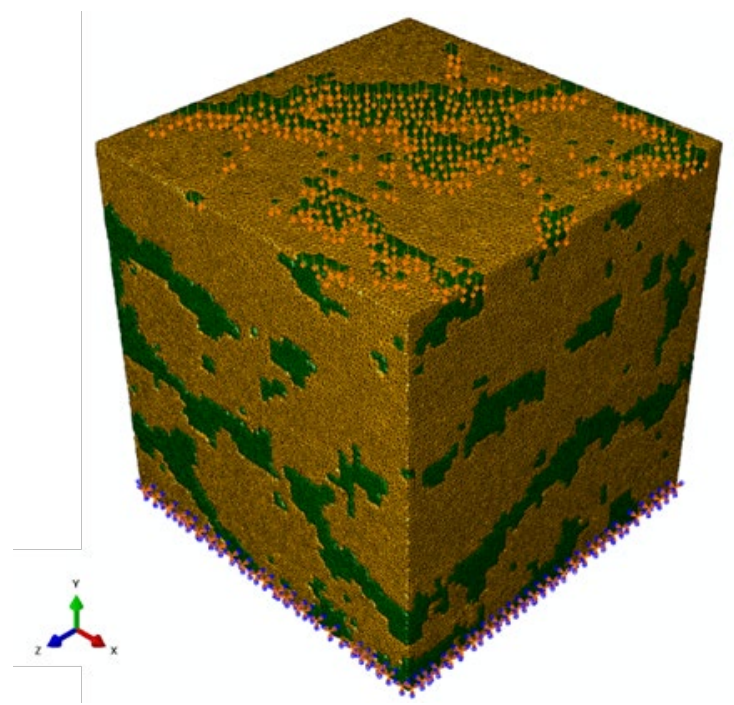

Figure 3 : Boundary conditions for RVE of bone sample. Yellow elements represent bone marrow and green represents the bone tissue. Orange arrows represent locations of the applied load (top distal surface), while the translation and rotation of the nodes on the bottom proximal surface were restricted

\subsection{Statistical analysis}

To evaluate the statistical significance $(p<0.05)$ of anatomic variations of the morphological and mechanical parameters of trabeculae in the studied distal tibia, Repeated Measures ANOVA (RM-ANOVA) tests were performed using SPSS Statistics 20.0 software (IBM Corp., NY, USA). The relationships between HR-pQCT measured local morphological parameters and the respective mechanical competence (stiffness) of trabecular RVE at five anatomic regions were evaluated using Pearson correlation coefficients $(r)$.

Furthermore, a stepwise multi-linear regression analysis was performed to predict the regional stiffness ( $\mathrm{k}_{\mathrm{A}}, \mathrm{k}_{\mathrm{P}}, \mathrm{kL}_{\mathrm{L}}, \mathrm{k}_{\mathrm{M}}, \mathrm{k}_{\mathrm{c}}$; subscripts denote respective regions) using directly 
measured local morphological parameters. Parameters which were significantly correlated with stiffness, such as BV/TV, Tb.Th, Tb.N, Tb.BS and Tb.Sp, were used as independent predictors during the regression analysis. At each step, one eligible independent variable with the highest statistic strength was selected in the predictive model. The process continued until no eligible independent predictor exceeded a statistic strength of 0.05 . The identified predictive local microstructural parameters were selected to yield the prediction of regional stiffness.

To test the precision of parameters measured in this study, existing reproducibility data sets from Paggiosi et al. [42] were retrospectively analysed. Two repeated scans of each participant were taken on the same day. Cubes were extracted using the same method as in this study on the common regions for two repeated scans. All directly measured trabecular parameters used in this study were calculated using a root mean square coefficient of variance (RMSCV) to determine the short-term reproducibility of measurements. The RMSCV of all regional trabecular measurements were with between $1 \%$ to $3.4 \%$, confirming good reliability of the methodology adopted in current study.

\section{Results}

\subsection{Regional variations of trabecular stiffness and microstructure}

The distribution of microstructural features and mechanical competence of trabecular bone differed significantly between five anatomic regions (anterior, posterior, lateral, medial and centre) of the studied distal tibiae $(p<0.05)$, as presented in Tables 2 and Figure 4. Local stiffness values obtained with finite-element analysis were significantly different $(p<0.05)$ between five anatomic regions (Table 2$)$ : the stiffness value of 
RVEs from medial $(9128.6 \mathrm{~N} / \mathrm{mm})$ region was significantly higher than those from the anterior $(2657.6 \mathrm{~N} / \mathrm{mm})$ and centre $(2015.7 \mathrm{~N} / \mathrm{mm})$ regions. In another words, anterior and centre region have the lowest stiffness among all the regions, with no significant difference between them.

Table 2: Statistics of mechanical stiffness of trabecular bone at different anatomic regions (A: anterior; P: posterior; L: Lateral; M: medial; C: centre)

\begin{tabular}{|c|c|c|c|c|c|}
\hline \multirow[t]{2}{*}{$\begin{array}{l}\text { Stiffness } \\
(\mathrm{N} / \mathrm{mm})\end{array}$} & \multirow[t]{2}{*}{ Mean } & \multirow[t]{2}{*}{$S D$} & \multicolumn{2}{|c|}{ 95\% Confidence Interval } & \multirow[t]{2}{*}{ Comparison } \\
\hline & & & $\begin{array}{l}\text { lower } \\
\text { bound }\end{array}$ & $\begin{array}{l}\text { Upper } \\
\text { bound }\end{array}$ & \\
\hline $\mathrm{kA}_{\mathrm{A}}$ & 2657.6 & 506.2 & 1589.6 & 3725.7 & $A<M^{*}$ \\
\hline $\mathrm{k}_{\mathrm{L}}$ & 5216.7 & 647.8 & 3849.9 & 6583.5 & $\mathrm{~L}>\mathrm{C}^{\dagger}$ \\
\hline $\mathrm{K}_{\mathrm{P}}$ & 7606.3 & 1060.6 & 5368.5 & 9844.0 & $\mathrm{P}>\mathrm{C}^{+}$ \\
\hline $\mathrm{k}_{\mathrm{M}}$ & 9128.6 & 1207.0 & 6582.1 & 11675.1 & $M>A^{*}, C^{*}$ \\
\hline $\mathrm{kc}_{\mathrm{c}}$ & 2015.7 & 411.1 & 1148.3 & 2883.1 & $\mathrm{C}<\mathrm{L}^{\dagger}, \mathrm{P}^{\dagger}, \mathrm{M}^{*}$ \\
\hline
\end{tabular}

Similar regional differences in trabecular volume fraction, surface area and plate-like structure fraction as for stiffness were observed (Table 3). RVEs from posterior and medial regions had significantly higher values of BV/TV, Tb.BS and Tb.N than those of the anterior and centre regions $(p<0.05)$. RVEs from the medial region had the highest trabecular volume fraction, trabecular thickness, trabecular surface area and plate-like structure fraction, significantly higher than those of anterior and central regions in BV/TV $(52.8 \%, 106 \%)$, Tb.Th $(12.3 \%, 12.3 \%)$, Tb.N $(20.98 \%, 30.04 \%)$, Tb.BS (47.2\%, 88.8\%) and PTb.N/TTb.N (36.2\%, 98.4\%), respectively. On the other hand, values of BV/TV, Tb.N, and Tb.BS from RVEs at anterior region were lowest among all regions except centre. The rankings of Tb.N and Tb.Sp among regions were almost in reverse order. The posterior segment had the highest Tb.N and lowest Tb.Sp , indicating a densely packed trabecular structure; while the anterior and centre were the most porous bone regions, with $20.3 \%$ and $25.8 \%$ lower Tb.N and $27.5 \%$ and $19.4 \%$ higher Tb.Sp than those of the posterior region, respectively. The medial 
region had the thickest trabeculae with the highest Tb.Th $(0.338 \mathrm{~mm})$. No significant differences were found between RVEs from posterior and medial regions for all the microstructural parameters $(p>0.05)$.

Table 3: Statistics of local and global morphological parameters of trabecular bone at different anatomic regions: A: anterior $(n=32)$; P: posterior $(n=32)$; L: Lateral $(n=32)$; M: medial $(n=32)$; C: centre $(n=8)$ and G: global $(n=4)$

\begin{tabular}{|c|c|c|c|c|c|c|}
\hline \multirow[t]{2}{*}{ Index } & \multirow[t]{2}{*}{ Region } & \multirow[t]{2}{*}{ Mean } & \multirow[t]{2}{*}{$S D$} & \multicolumn{2}{|c|}{$\begin{array}{r}\text { 95\% Confidence } \\
\text { Interval }\end{array}$} & \multirow[t]{2}{*}{ Comparison } \\
\hline & & & & $\begin{array}{l}\text { lower } \\
\text { bound }\end{array}$ & $\begin{array}{l}\text { Upper } \\
\text { bound }\end{array}$ & \\
\hline $\mathrm{BV} / \mathrm{TV}_{\mathrm{A}}$ & $\mathrm{A}$ & 0.197 & 0.012 & 0.172 & 0.222 & $A<M^{*}, A>C^{*}$ \\
\hline $\mathrm{BV} / \mathrm{TV} \mathrm{L}$ & $\mathrm{L}$ & 0.243 & 0.015 & 0.212 & 0.275 & $L>C \ddagger$ \\
\hline $\mathrm{BV} / \mathrm{TV} \mathrm{P}_{\mathrm{P}}$ & $P$ & 0.271 & 0.018 & 0.233 & 0.310 & $P>C^{\ddagger}$ \\
\hline $\mathrm{BV} / \mathrm{TV}_{\mathrm{M}}$ & $M$ & 0.301 & 0.020 & 0.259 & 0.343 & $M>A^{*}, C^{*}$ \\
\hline $\mathrm{BV} / \mathrm{TV} \mathrm{c}$ & C & 0.146 & 0.011 & 0.123 & 0.170 & $C<A^{*}, P^{*}, L^{*}, M^{*}$ \\
\hline $\mathrm{BV} / \mathrm{TV}^{\mathrm{d}}$ & G & 0.109 & 0.016 & 0.094 & 0.133 & \\
\hline $\mathrm{Tb} \mathrm{Th}_{\mathrm{A}}(\mathrm{mm})$ & $A$ & 0.301 & 0.005 & 0.290 & 0.312 & $A<M^{*}$ \\
\hline Tb.Thь & L & 0.297 & 0.006 & 0.284 & 0.309 & $\mathrm{~L}<\mathrm{M}^{*}$ \\
\hline Tb.Thp & $P$ & 0.311 & 0.008 & 0.294 & 0.329 & \\
\hline Tb.ThM & $M$ & 0.338 & 0.007 & 0.322 & 0.353 & $M>A^{*}, L^{*}$ \\
\hline Tb.Thc & $\mathrm{C}$ & 0.301 & 0.005 & 0.290 & 0.312 & $C<M^{*}$ \\
\hline Tb.Th ${ }^{d}$ & G & 0.063 & 0.008 & 0.036 & 0.091 & \\
\hline Tb. $\mathrm{N}_{\mathrm{A}}(1 / \mathrm{mm})$ & A & 1.349 & 0.033 & 1.278 & 1.419 & $\mathrm{~A}<\mathrm{M}^{\dagger}, \mathrm{P}^{\dagger}, \mathrm{L}^{\dagger}$ \\
\hline Tb. $\mathrm{N}_{\mathrm{L}}$ & L & 1.636 & 0.044 & 1.542 & 1.729 & $L>A^{*}, C^{*}$ \\
\hline Tb. $\mathrm{N}_{\mathrm{P}}$ & $P$ & 1.692 & 0.049 & 1.588 & 1.796 & $P>A^{*}, C^{*}$ \\
\hline Tb. $\mathrm{N}_{M}$ & $M$ & 1.632 & 0.065 & 1.495 & 1.769 & $M>A^{*}, C^{*}$ \\
\hline Tb.Nc & C & 1.255 & 0.38 & 1.176 & 1.335 & $C<P^{*}, L^{*}, M^{*}$ \\
\hline Tb.N d & $\mathrm{G}$ & 1.720 & 0.129 & 1.540 & 1.870 & \\
\hline $\mathrm{Tb} \mathrm{BS}_{\mathrm{A}}\left(\mathrm{mm}^{2}\right)$ & A & 117.277 & 6.260 & 104.069 & 130.485 & $\mathrm{~A}<\mathrm{L}^{\dagger}, \mathrm{M}^{\dagger}, \mathrm{A}>\mathrm{C}^{\ddagger}$ \\
\hline Tb.BSL & L & 150.018 & 8.300 & 132.506 & 167.529 & $L>A^{\dagger}, C^{*}$ \\
\hline Tb.BSP & $P$ & 151.952 & 8.284 & 134.474 & 169.430 & $\mathrm{P}>\mathrm{C}^{*}$ \\
\hline Tb.BS ${ }_{M}$ & $M$ & 172.608 & 8.887 & 153.857 & 191.359 & $M>A^{\dagger}, C^{*}$ \\
\hline Tb.BSc & C & 91.430 & 6.616 & 77.472 & 105.387 & $C<A^{\ddagger}, P^{*}, L^{*}, M^{*}$ \\
\hline $\mathrm{Tb} \mathrm{Sp}_{\mathrm{A}}(\mathrm{mm})$ & $A$ & 0.756 & 0.018 & 0.718 & 0.794 & $\mathrm{~A}>\mathrm{M}^{\dagger}, \mathrm{P}^{\dagger}, \mathrm{L}^{\dagger}$ \\
\hline $\mathrm{Tb} . \mathrm{Sp}_{\mathrm{L}}$ & L & 0.632 & 0.015 & 0.600 & 0.665 & $\mathrm{~L}<\mathrm{A}^{\dagger}, \mathrm{C}^{*}$ \\
\hline Tb.Spp & $P$ & 0.633 & 0.014 & 0.602 & 0.663 & $P<A^{\dagger}, C^{*}$ \\
\hline Tb.SpM & $M$ & 0.635 & 0.022 & 0.587 & 0.682 & $\mathrm{M}<\mathrm{A}^{\dagger}, \mathrm{C}^{*}$ \\
\hline Tb.Spc & C & 0.807 & 0.025 & 0.753 & 0.861 & $C<P^{*}, L^{*}, M^{*}$ \\
\hline Tb.Spd & G & 0.522 & 0.046 & 0.429 & 0.615 & \\
\hline $\mathrm{PTb} . \mathrm{N} / \mathrm{T} T \mathrm{~Tb} . \mathrm{N}_{\mathrm{A}}$ & A & 0.459 & 0.036 & 0.383 & 0.536 & $A<M^{*}, A>C^{\ddagger}$ \\
\hline $\mathrm{PTb} . \mathrm{N} / \mathrm{T}$ Tb.N $\mathrm{N}_{\mathrm{L}}$ & L & 0.496 & 0.039 & 0.413 & 0.579 & $L>C \ddagger$ \\
\hline $\mathrm{PTb} . \mathrm{N} / \mathrm{T}$ Tb. $\mathrm{N}_{\mathrm{P}}$ & $\mathrm{P}$ & 0.533 & 0.035 & 0.460 & 0.607 & $P>C \ddagger$ \\
\hline $\mathrm{PTb} . \mathrm{N} / \mathrm{T}$ Tb. $\mathrm{N}_{\mathrm{M}}$ & M & 0.625 & 0.039 & 0.542 & 0.708 & $M>A^{*}, C^{*}$ \\
\hline $\mathrm{PTb} . \mathrm{N} / \mathrm{T} T b . \mathrm{N}_{\mathrm{c}}$ & C & 0.315 & 0.040 & 0.230 & 0.401 & $C<A^{\ddagger}, P^{\ddagger}, L^{\ddagger}, M^{*}$ \\
\hline
\end{tabular}

' $d$ ' indicates parameters derived indirectly from other known parameters.

${ }^{*} p<0.001$

$+p<0.01$

$\ddagger p<0.05$ 

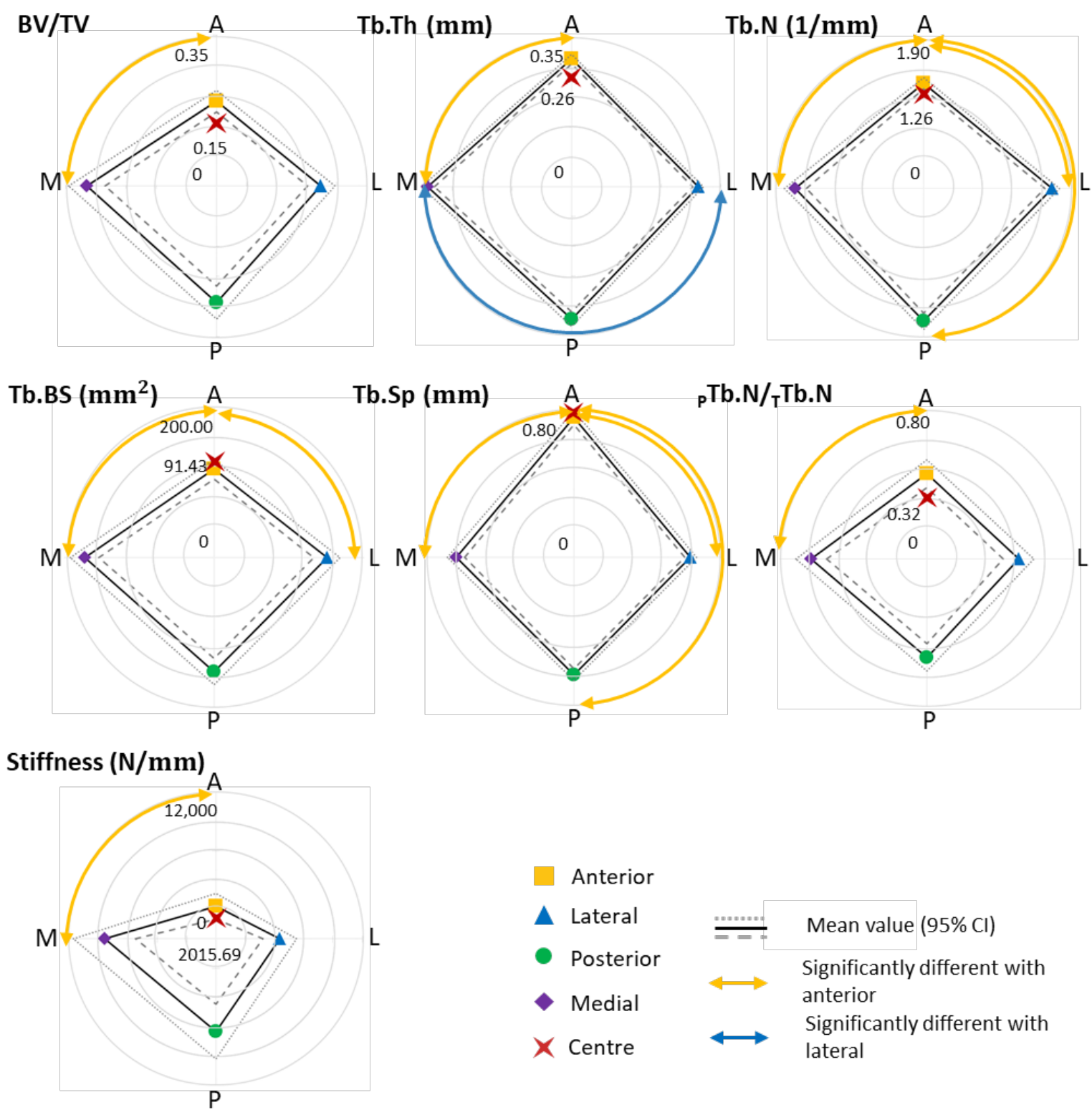

Figure 4: Microstructural parameters and mechanical competence of trabecular bone for anatomic regions: A (anterior), P (posterior), L (Lateral), M (medial) and C (centre) of distal tibia: BV/TV, Tb.Th, Tb.N, Tb.BS, Tb.Sp, PTb.N/TTb.N, and stiffness; mean (black solid line) values $\pm 95 \% \mathrm{Cl}$ are given in each graph. Significant differences between regions were illustrated and linked with double-sided arrows. Anterior and lateral regions demonstrated consistent statistical significance with respect to other regions.

In comparison to the local trabecular morphological parameters, the results from the global derived assessment (average value of the entire trabecular scanned area) revealed that $\mathrm{BV} / \mathrm{TV}^{\mathrm{d}}$ and $\mathrm{Tb}^{\mathrm{T}} \mathrm{Th}^{\mathrm{d}}$ (average 0.109 and $0.063 \mathrm{~mm}$ ) were considerably lower than their local counterparts, consistent with reports that derived parameters were significantly underestimated [43]. BV/TV ranged from $33.9 \%$ in the centre region 
to $176.1 \%$ higher in the medial region relative to the global value, and $\mathrm{Tb}$.Th in the medial region was five times higher than global mean. However, the standard global measure, showed similar values in Tb.N (average 1.72/mm) compared with local analysis of $1.69 / \mathrm{mm}$ at posterior region. These estimated global and local parameters of the trabecular bone were within the ranges reported elsewhere [19][21][27].

\subsection{Regional correlation between microstructure and stiffness}

There were strong and consistent correlations $(p<0.01)$ between region-specific trabecular stiffness and all of the analysed microstructural parameters in four anatomic regions, excepting the centre region (Figure 5). Among the local microstructural parameters, the strongest correlation of stiffness value of a given region was always within respective local regions; for instance, the correlation coefficients between stiffness in the anterior region and respective local microstructural parameters, BV/TV $\mathrm{A}$ $(r=0.92), \operatorname{Tb} . \operatorname{Th}_{A}(r=0.79), \operatorname{Tb} . N_{A}(r=0.78), \operatorname{Tb}_{B} B S_{A}(r=0.84), \operatorname{Tb} . \operatorname{Sp}_{A}(r=0.71)$, $\mathrm{PTb} . \mathrm{N} / \mathrm{TTb} . \mathrm{N}_{\mathrm{A}}(\mathrm{r}=0.78)$ were the highest when compared to those from other regions. Trabecular volume fraction was the strongest correlate of stiffness in all regions, followed by Tb.BS. The values of correlation coefficient of BV/TV were consistently high and varied little among the regions, while $\mathrm{PTb} . \mathrm{N} / \mathrm{T}$ Tb.N had the lowest correlation with stiffness across all regions. The correlations between regional morphology and regional stiffness were generally higher than the correlations between global morphology and global stiffness observed in this study ( $r=0.36$ to 0.62$)$ as well as the correlations previously reported that varied from 0.5 to 0.9 [44] (Fig. 5). The results of a linear correlation analysis (presented in Appendix) between the studied anatomical regions also confirmed that morphology parameters between the anterior and 
posterior regions were significantly correlated; this was also true for the medial and lateral regions.

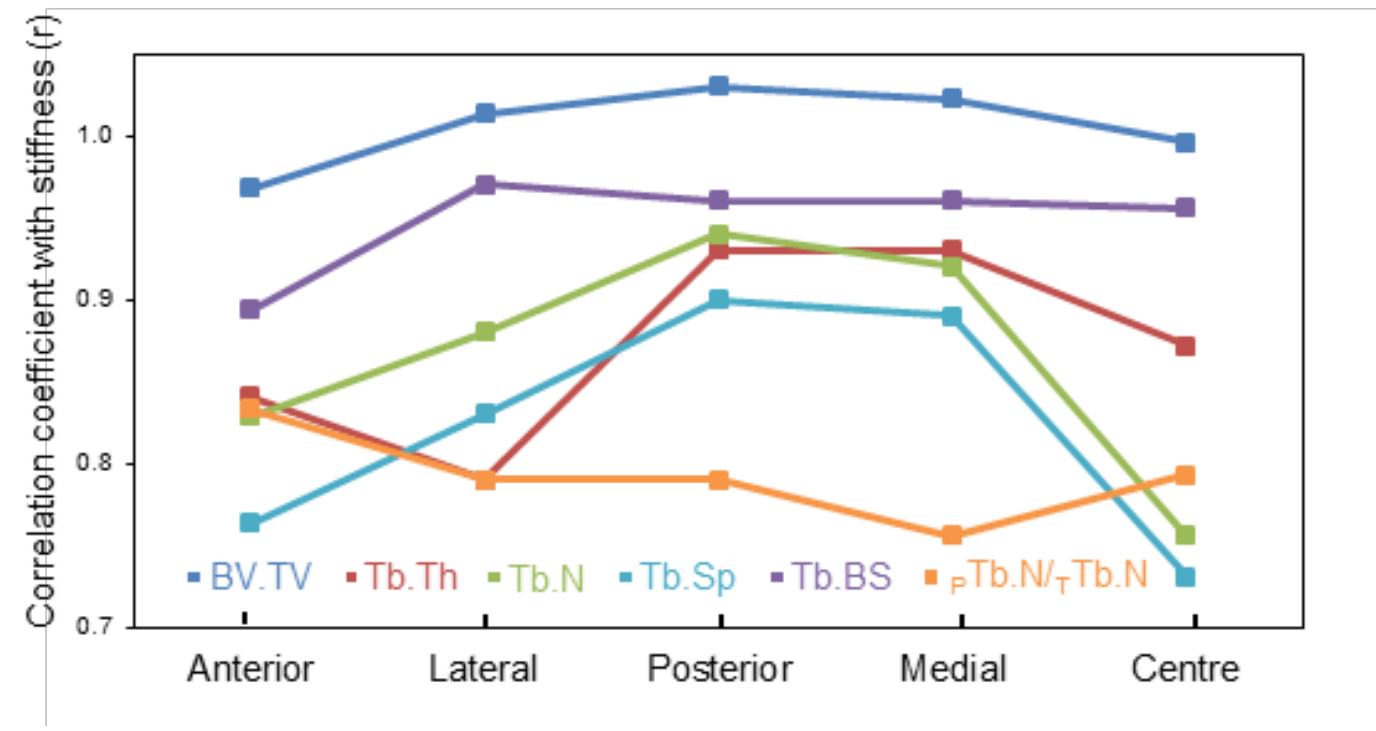

Figure 5: Correlation coefficients ( $r$ ) of regional (square) stiffness with microstructural parameters.

\subsection{Predictive power of morphological parameters to stiffness}

Stepwise multi-linear regression between stiffness from each region with their respective local morphological parameters was calculated to determine which parameters were most predictive of regional trabecular stiffness (Table 4). The trabecular volume fraction was consistently the strongest significant independent predictor for all regions; whilst additional independent predictors contributed at some regions. The results of predictive models showing relationship between regional stiffness value and respective independent predictor(s) are fitted with experimental data and shown in Figure 6, with strong adjusted R-square, $\mathrm{k}_{\mathrm{A}}\left(\mathrm{r}^{2}=0.921\right), \mathrm{kP}_{\mathrm{P}}\left(\mathrm{r}^{2}=\right.$ 0.959), $k L\left(r^{2}=0.949\right), k_{M}\left(r^{2}=0.955\right)$ and $k c\left(r^{2}=0.890\right)$. Multi-regression equations were also calculated for the prediction model of each region. 
Table 4: Coefficient of determination $\left(R^{2}\right)$ and independent predictors of multi-linear regression analysis for predicting regional stiffness through respective morphology parameters (A: anterior; P: posterior; L: Lateral; M: medial; C: centre)

\begin{tabular}{|c|c|c|c|}
\hline $\begin{array}{l}\text { Mechanical } \\
\text { properties }\end{array}$ & $\begin{array}{l}\text { Variables entering multi-linear regression } \\
\text { model }\end{array}$ & $\mathrm{R}^{2}$ & Adjusted $\mathrm{R}^{2}$ \\
\hline $\mathrm{k}_{\mathrm{A}}$ & BV/TV ${ }^{\ddagger}{ }^{\ddagger}, T b . S p_{A}, T b . B S_{A} T b . T h_{A}, P T b . N / T T b . N_{A} \ddagger$ & 0.926 & 0.921 \\
\hline $\mathrm{k}_{\mathrm{P}}$ & $\mathrm{BV} / \mathrm{TV}_{\mathrm{p}}{ }^{*}, \mathrm{~Tb} . \mathrm{Sp}_{\mathrm{p}} \ddagger$ & 0.960 & 0.959 \\
\hline $\mathrm{kL}$ & BV/TV $L^{*}$, PTb.N/TTb.NL ${ }^{\ddagger}, T b . S p L$ Tb.Th ${ }^{\ddagger}$ & 0.952 & 0.949 \\
\hline $\mathrm{k}_{\mathrm{M}}$ & BV/TV ${ }^{*}$, Tb.SpM, Tb. $\mathrm{N}_{\mathrm{M}}$ & 0.957 & 0.955 \\
\hline $\mathrm{k}_{\mathrm{c}}$ & $\mathrm{BV} / \mathrm{TV}_{\mathrm{C}}{ }^{*}$ & 0.895 & 0.890 \\
\hline
\end{tabular}

${ }^{*} p<0.001$

$\mp p<0.05$ 

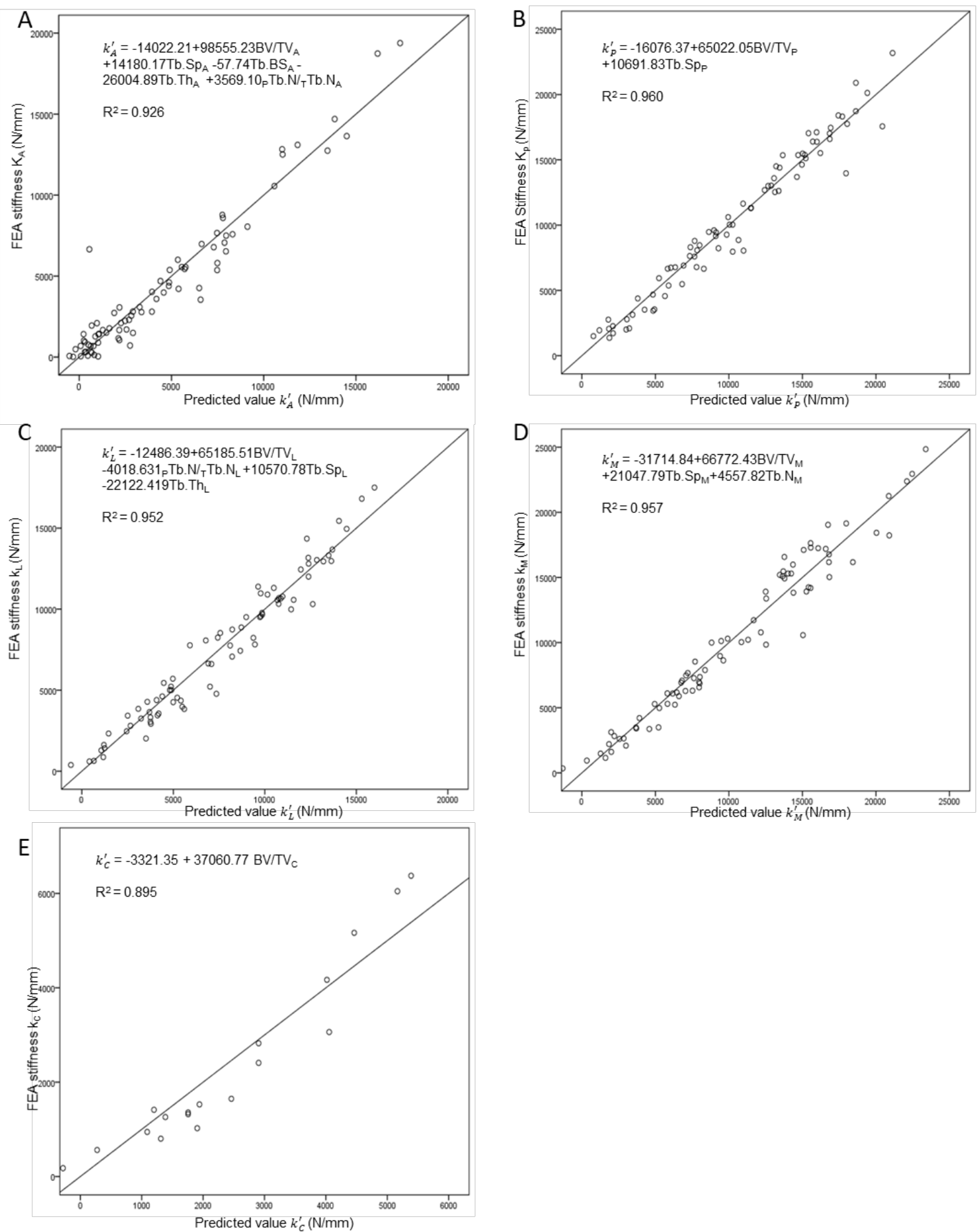

Figure 6: Results of multi-linear regression analyses between regional stiffness $(k)$ and relevant predictors of microstructural parameters (k') in Anterior (A), Posterior (B), Lateral (C), Medial (D) and Centre (E) regions. 


\section{Discussion}

The results of the study highlighted for the first time the implications for bone stiffness estimated from a finite element model, assessed in-vivo using HR-pQCT of human distal tibia, with stiffness being 4.5 times higher in the highest (medial) than lowest (central) region. Significant regional variation in trabecular microarchitecture was measured, with up to $100 \%$ differences between lowest (central and anterior) and highest (medial and posterior) regions. BV/TV was considerably higher (33.9-176.1\%) at all the regions in regional analysis than in global analysis, with similar patterns also for Tb.Th. A novel finding was the higher proportion of plate-like trabecular morphology in medial and posterior regions. Bone volume fraction was the strongest predictor of stiffness across all regions: the respective correlation coefficient could be up to $55 \%$ higher than that of the global value.

The observed microstructural distribution indicates that the medial and posterior regions were, consistently among all participants, significantly denser, thicker, and "stronger" than other regions. Parameters such as BV/TV, Tb.Th and Tb.BS in values at the highest (medial) region $52.8 \%, 12.3 \%$ and $47.2 \%$ higher than the lowest (anterior) region. Comparing with the global mean value, Tb.Th in the medial region was five times higher and BV/TV varied from $33.9 \%$ higher in the centre region to $176.1 \%$ higher in the medial region. This regional variation and difference to the global measurement were consistent with a previous study [27]. Possible reasons for the difference in value between local and global parameters are: (1) measurement across the entire trabecular compartment reduces the global mean value when significant variation and material heterogeneity exist locally; (2) the global analysis used a standard HR-pQCT protocol (derived measurement), while the regional analysis 
employed a widely adopted direct-imaging-analysis protocol implemented in BoneJ [31]. In an early study, Liu et al. [43] made comparison between the two techniques and they concluded that both methods correlated well with respect to their gold standards. Still, they reported that HR-pQCT derived-global parameters considerably underestimated the values obtained compared to direct measurement, consistent with the findings from this study. The authors further suggested that this underestimation might be due to the global derived technique employed in the HR-pQCT patient evaluation protocol, which could be improved by an advanced image-processing method [45].

The differences in proportion of plate-like and rod-like structures could affect mechanical competence of trabecular bone [43][46][47] and may be associated with postmenopausal osteoporosis [44][48]. Comparative studies of plate-like and rod-like structures were previously conducted between different parts of bones, between healthy and diseased bones [49] and between different ethnicities/races [50][51]. However, their spatial distribution among anatomical regions of the same bone is still not fully understood. In this study, we analysed regional variation in plate-like structure fraction $\mathrm{PTb} . \mathrm{N} / \mathrm{TTb} . \mathrm{N}$ and found that the medial and posterior regions were dominated by plate-like structures, with the average value of PTb.N/TTb.N higher than $50 \%$. Compared with the global average of plate volume to bone volume ratio - PV/BV $(0.373)$ of the distal radius from Pialat et al. [47], the regional plate-like structure fractions in the current study were higher for all but centre region. This discrepancy is likely attributed to the difference of trabecular micro-morphology between these two regions: the distal tibia tends to have more plate-like structure than distal radius [52]. It was also reported that tibial trabecular compartment carries up to $71 \%$ of axial loads at the distal section [53]. During daily activities, loading magnitudes and modes differ from 
site to site [54], particularly for the distal tibia where large compressive and shear forces are imposed on the medial and posterior regions [55][56]. These regional differences observed in this study and elsewhere [27][54] maybe caused by spatial non-uniformity of mechanical loading as explained by Wolff's law. Furthermore, physical activity, pain or pathological conditions may differentially affect loading at different regions, contributing to regional variation [57]. Conversely, exercise could be used to target adaptation at regions of low mechanical competence.

Previous studies have demonstrated spatial variability of trabecular bone microstructure as well as the effect of gender, age, drugs and osteoporosis [27][28][58]. There is, however, a lack of understanding of the regional variation of trabecular stiffness, which correlates significantly with fracture resistance estimated from HRpQCT-based FEA analysis [43]. Our results showed that the medial region exhibited the highest stiffness, consistent with the microstructure measurements. The differences in stiffness between medial and anterior, medial and centre regions were 3.4 times and 4.5 times higher, respectively. Our results also confirmed that the regionspecific trabecular stiffness was significantly correlated with respective local morphological parameters. Regional microarchitectural parameters and stiffness were more highly correlated than global correlations (from different cohort) reported in the literature [44]. We conjecture that regional analyses may have the potential to provide a more reliable method in prediction of trabecular stiffness.

With regional variations of the microstructure varying more than $100 \%$, we hypothesise that the predictive models for stiffness may vary between anatomic regions. This hypothesis was supported by the results of multi-linear regression: whilst BV/TV contributed independently to stiffness at all regions, trabecular spacing was also a 
strong predictor at all but centre region with other parameters contributing selectively at different regions. By employing the multiple microstructural parameters, a strong predictive ability was shown, indicating that not only bone volume but also trabecular architecture resulting from bone adaptation may contribute to mechanical competence [59], which may allow them to function as indicators of fracture risk and highlight the importance of examining structural parameters in addition to bone volume alone.

Previous study has shown that standard HR-pQCT indices and morphological assessment of the peripheral skeleton improved prediction of fracture risk beyond femoral neck areal bone mineral density (aBMD) or the Fracture Risk Assessment Tool (FRAX) scores alone [60]. One possible implication of our finding is that regional variation of trabecular microarchitecture and mechanical competence has the potential to improve the understanding of fracture location at distal tibia. Topliss et al. [61] investigated the fracture patterns of the tibia pilon fracture and reported that more than $60 \%$ of fractures pass along the antero-lateral and postero-lateral directions, consistent with the $\mathrm{Y}$-type and $\mathrm{V}$-type which are the most common fracture patterns observed. The current study demonstrated that morphological and mechanical properties were worse in anterior and lateral than other regions, with thinner and looser structures with lower stiffness. So, one possible scenario is that, under uniform loading conditions, anterior and lateral regions are more prone to fracture. Furthermore, identification of regional defects could help to identify and evaluate possible countermeasures such as exercise, and potentially help to design region specific interventions.

There are several limitations in this study. First, the number of samples for each anatomic region is limited to eighty, extracted from ten participants. However, the 
overall segmented area using RVEs covers the majority of the cross-section of the distal tibia and the statistical difference among anatomical regions were significant. A large effect size (0.8) was calculated alongside the significant difference, demonstrating that the number of subjects in this study was sufficient to ensure that the study had acceptable power [62]. Second, the implication of direct comparison between regional and global measurements from other research is limited due to differences in sample size and origin, and algorithms used to calculate morphological parameters. Furthermore, because of the high requirement of computational power, the direct measurement method can only be used to assess the microstructure of subvolumes extracted from the five anatomic regions rather than measuring the entire region of each region. To examine the size effect for subvolume, different RVE side lengths were used and no major changes in results were observed for RVEs with length in excess of $4 \mathrm{~mm}$. In addition, this study is focused on micro-morphology analysis of trabecular bone; distinguishing between the cortical and trabecular bone was conducted manually following standard protocol [63] to ensure no cortical bone was selected, therefore, transitional or cortico-trabecular junctional zone was not considered in the current study. Last but not least, the spatial resolution of HR-pQCT images was limited to $82 \mu \mathrm{m}$ (voxel size) due to the capacity of the scanner; so, structural feature below this threshold may not be detectable. However, the technique can still provide highly correlated results against the gold-standard technique for monitoring relative (regional) variation of trabecular microstructure [52].

\section{Conclusion}

In conclusion, the in-vivo image based structural-function assessment presented in this study demonstrated that the marked regional variation in trabecular mechanical 
competence is associated with regional variation in microarchitecture of the trabecular bone. Regional morphological parameters were consistently highly correlated with mechanical competence of trabecular bone at all regions studied, with regional BV/TV being the strongest predictor. The method developed for regional analysis of trabecular mechanical competence may offer an insight into the relationship between mechanical behaviour and microstructure of bone, which might not be noticeable in global analysis as a result of excessive averaging over an entire trabecular compartment. The findings have implications for the use of in-vivo HR-pQCT for detection and study of bone degenerative diseases: quantification of regional variations in stiffness and trabecular microstructure may allow better understanding of fracture risk in conjunction to other co-contributing factors.

\section{Acknowledgement}

The authors would like to thank all participants involved in this study as well as Ganggang Xiang and Yaqi Qian from Loughborough University, for their contribution in segmenting and analysing the parameters. We would also like to acknowledge the Mellanby Centre for Bone Research at The University of Sheffield and the NIHR Clinical Research Facility, Sheffield Teaching Hospitals for providing access to the HR-pQCT scanner.

\section{Funding}

This study was jointly supported by Loughborough University Health \& Wellbeing Research Challenge Seedcorn fund and NIHR Leicester Biomedical Research Centre. 


\section{Disclosure}

The authors declare that there are no conflicts of interest.

\section{Authors' roles}

Study design: SL and JD. Study conduct: JD, $\mathrm{CH}$ and MAP. Data collection: JD, CH, MAP and JW. Data analysis: JD and MAP. Data interpretation: JD, SL, KBW, VVS. Drafting manuscript: JD. Revising manuscript content: JD, SL, KBW and VVS. Approving final version of manuscript: all authors.

\section{Reference}

1. Kanis J. Diagnosis of osteoporosis and assessment of fracture risk. Lancet . 2002;359(9321), 1929-1936.

2. Consensus development conference: Diagnosis, prophylaxis, and treatment of osteoporosis. Am J Med. Elsevier; 1993 Jun 1;94(6), 646-50.

3. Nikander R., Sievänen H., Heinonen A., Daly R.M., Uusi-rasi K., Kannus P. Targeted exercise against osteoporosis : A systematic review and meta-analysis for optimising bone strength throughout life. 2010;

4. Ascenzi M.G., Hetzer N., Lomovtsev A., Rude R., Nattiv A., Favia A. Variation of trabecular architecture in proximal femur of postmenopausal women. $J$ Biomech. Elsevier; 2011;44(2), 248-56.

5. Macdonald H.M., Cooper D.M.L., McKay H.A. Anterior-posterior bending strength at the tibial shaft increases with physical activity in boys: Evidence for 
non-uniform geometric adaptation. Osteoporos Int. 2009;20(1), 61-70.

6. Miller P.D., Bonnick S.L., Rosen C.J., Altman R.D., Avioli L. V, Dequeker J., et al. Clinical utility of bone mass measurements in adults: consensus of an international panel. The Society for Clinical Densitometry. Semin Arthritis Rheum. 1996 Jun;25(6), 361-72.

7. Beck T.J., Looker A.C., Ruff C.B., Sievanen H., Wahner H.W. Structural Trends in the Aging Femoral Neck and Proximal Shaft: Analysis of the Third National Health and Nutrition Examination Survey Dual-Energy X-Ray Absorptiometry Data. J Bone Miner Res. 2000 Dec 1;15(12), 2297-304.

8. Ma H., Leskinen T., Alen M., Cheng S., Sipilä S., Heinonen A., et al. Long-term leisure time physical activity and properties of bone: a twin study. $J$ Bone Miner Res. 2009;24(8), 1427-33.

9. Shaw C.N., Stock J.T. Intensity, repetitiveness, and directionality of habitual adolescent mobility patterns influence the tibial diaphysis morphology of athletes. Am J Phys Anthropol. 2009;140(1), 149-59.

10. Rantalainen T., Nikander R., Heinonen A., Suominen H., Sievänen H. Directionspecific diaphyseal geometry and mineral mass distribution of tibia and fibula: A pQCT study of female athletes representing different exercise loading types. Calcif Tissue Int. 2010;86(6), 447-54.

11. Li S., Denirci E., Silberschmidt V. V. Variability and anisotropy of mechanical behavior of cortical bone in tension and compression. J Mech Behav Biomed Mater. 21, 109-20. 
12. Eastell R., Walsh J.S. Bone: Microarchitecture of bone predicts fractures in older women. Nat Rev Endocrinol. 2018;14(5).

13. Yeni Y.N., Zinno M.J., Yerramshetty J.S., Zauel R., Fyhrie D.P. Variability of trabecular microstructure is age-, gender-, race- and anatomic site-dependent and affects stiffness and stress distribution properties of human vertebral cancellous bone. Bone. Elsevier Inc.; 2011;49(4), 886-94.

14. Milovanovic P., Djonic D., Marshall R.P., Hahn M., Nikolic S., Zivkovic V., et al. Micro-structural basis for particular vulnerability of the superolateral neck trabecular bone in the postmenopausal women with hip fractures. Bone. Elsevier Inc.; 2012;50(1), 63-8.

15. Zhou B., Liu X.S., Wang J., Lu X.L., Fields A.J., Guo X.E. Dependence of mechanical properties of trabecular bone on plate-rod microstructure determined by individual trabecula segmentation (ITS). J Biomech. 2014;47, 702-8.

16. Mueller T.L., van Lenthe G.H., Stauber M., Gratzke C., Eckstein F., Müller R. Regional, age and gender differences in architectural measures of bone quality and their correlation to bone mechanical competence in the human radius of an elderly population. Bone. Elsevier Inc.; 2009;45(5), 882-91.

17. Nishiyama K.K., Shane E. Clinical imaging of bone microarchitecture with HRpQCT. Curr Osteoporos Rep. 2013;11(2), 147-55.

18. Boutroy S., Bouxsein M.L., Munoz F., Delmas P.D. In vivo assessment of trabecular bone microarchitecture by high-resolution peripheral quantitative 
computed tomography. J Clin Endocrinol Metab. 2005;90(12), 6508-15.

19. Khosla S., Riggs L., Atkinson E.J., Oberg A.L., Mcdaniel L.J., Holets M., et al. Effects of Sex and Age on Bone Microstructure at the Ultradistal Radius: A Population-Based Noninvasive In Vivo Assessment. J Bone Min Res. 2006;21, $124-31$.

20. Dalzell N., Kaptoge S., Morris N., Berthier A., Koller B., Braak L., et al. Bone micro-architecture and determinants of strength in the radius and tibia: agerelated changes in a population-based study of normal adults measured with high-resolution pQCT. Osteoporos Int. 2009;20, 1683-94.

21. Burrows M., Liu D., McKay H. High-resolution peripheral QCT imaging of bone micro-structure in adolescents. Osteoporos Int. 2010;21(3), 515-20.

22. Chavassieux P., Karsdal M.A., Segovia-Silvestre T., Neutzsky-Wulff A. V, Chapurlat R., Boivin G., et al. Mechanisms of the Anabolic Effects of Teriparatide on Bone: Insight From the Treatment of a Patient With Pycnodysostosis. J Bone Miner Res. 2008;23(7), 1076-83.

23. Bacchetta J., Boutroy S., Vilayphiou N., Juillard L., Guebre-Egziabher F., Rognant N., et al. Erratum: Early impairment of trabecular microarchitecture assessed with HR-pQCT in patients with stage II-IV chronic kidney disease (Journal of Bone and Mineral Research (2010) 25:4 (849-57)). J Bone Miner Res. 2012;27(11), 2413.

24. Rubin M.R., Dempster D.W., Kohler T., Stauber M., Zhou H., Shane E., et al. Three dimensional cancellous bone structure in hypoparathyroidism. Bone. 
Elsevier Inc.; 2010;46(1), 190-5.

25. Varga P., Pahr D.H., Baumbach S., Zysset P.K. HR-pQCT based FE analysis of the most distal radius section provides an improved prediction of Colles' fracture load in vitro. Bone. Elsevier Inc.; 2010;47(5), 982-8.

26. Lai Y.M., Qin L., Yeung H.Y., Lee K.K.H., Chan K.M. Regional differences in trabecular BMD and micro-architecture of weight-bearing bone under habitual gait loading - A pQCT and microCT study in human cadavers. Bone. 2005;37(2), 274-82.

27. Sode M., Burghardt A.J., Kazakia G.J., Link T.M., Majumdar S. Regional variations of gender-specific and age-related differences in trabecular bone structure of the distal radius and tibia. Bone. Elsevier Inc.; 2010;46(6), 1652-60.

28. Burghardt A.J., Kazakia G.J., Sode M., De Papp A.E., Link T.M., Majumdar S. A longitudinal HR-pQCT study of alendronate treatment in postmenopausal women with low bone density: Relations among density, cortical and trabecular microarchitecture, biomechanics, and bone turnover. $J$ Bone Miner Res. 2010;25(12), 2282-95.

29. Pauchard Y., Liphardt A.M., Macdonald H.M., Hanley D.A., Boyd S.K. Quality control for bone quality parameters affected by subject motion in high-resolution peripheral quantitative computed tomography. Bone. Elsevier Inc.; 2012;50(6), $1304-10$.

30. Singh A.K., Lobo Gajiwala A., Rai R.K., Khan M.P., Singh C., Barbhuyan T., et al. Cross-correlative 3D micro-structural investigation of human bone processed 
into bone allografts. Mater Sci Eng C. 2016;62, 574-84.

31. Doube M., Kłosowski M.M., Arganda-Carreras I., Cordelières F.P., Dougherty R.P., Jackson J.S., et al. BoneJ: free and extensible bone image analysis in ImageJ.

32. Doube M., Salmon P. The ellipsoid factor for quantification of rods, plates, and intermediate forms in 3D geometries. 2015;

33. Hildebrand T., Rüegsegger P. A new method for the model-independent assessment of thickness in three-dimensional images. J Microsc. 1997;185(1), $67-75$.

34. Dougherty R.P., Kunzelmann K.-H. Computing Local Thickness of 3D Structures with ImageJ. 2017;

35. Lorensen W.E., Cline H.E. MARCHING CUBES: A HIGH RESOLUTION 3D SURFACE CONSTRUCTION ALGORITHM. Comput Graph. 1987;21(4).

36. Laib A., Häuselmann H.J., Rüegsegger P. In vivo high resolution 3D-QCT of the human forearm. Technol Health Care. 1998 Dec;6(5-6), 329-37.

37. Uller R., Van Campenhout H., Van Damme B., Van Der Perre G., Dequeker J., Hildebrand T., et al. Morphometric Analysis of Human Bone Biopsies: A Quantitative Structural Comparison of Histological Sections and MicroComputed Tomography.

38. Klintström E., Klintström B., Moreno R., Brismar T.B., Pahr D.H., Smedby Ö. Predicting Trabecular Bone Stiffness from Clinical Cone-Beam CT and HR- 
pQCT Data; an In Vitro Study Using Finite Element Analysis. PLoS One. 2016;11(8).

39. Joldes G.R., Doyle B., Wittek A., Nielsen P.M.F., Miller K., editors. Computational Biomechanics for Medicine [Internet]. Cham: Springer International Publishing; 2016.

40. MacNeil J.A., Boyd S.K. Bone strength at the distal radius can be estimated from high-resolution peripheral quantitative computed tomography and the finite element method. Bone. 2008;42(6), 1203-13.

41. Chen Y., Pani M., Taddei F., Mazzà C., Li X., Viceconti M. Large-Scale Finite Element Analysis of Human Cancellous Bone Tissue Micro Computer Tomography Data: A Convergence Study. J Biomech Eng. 2014;136(10), 101013.

42. Paggiosi M.A., Eastell R., Walsh J.S. Precision of high-resolution peripheral quantitative computed tomography measurement variables: Influence of gender, examination site, and age. Calcif Tissue Int. 2014;94(2), 191-201.

43. Liu X.S., Zhang X.H., Sekhon K.K., Adam M.F., McMahon D.J., Bilezikian J.P., et al. High-Resolution Peripheral Quantitative Computed Tomography Can Assess Microstructural and Mechanical Properties of Human Distal Tibial Bone. J Bone Miner Res. 2009;25(4), 090923075317031-48.

44. Liu X.S., Cohen A., Shane E., Yin P.T., Stein E.M., Rogers H., et al. Bone density, geometry, microstructure, and stiffness: Relationships between peripheral and central skeletal sites assessed by DXA, HR-pQCT, and cQCT in 
premenopausal women. J Bone Miner Res. 2010;25(10), 2229-38.

45. Burghardt A.J., Kazakia G.J., Majumdar S. A local adaptive threshold strategy for high resolution peripheral quantitative computed tomography of trabecular bone. Ann Biomed Eng. 2007;35(10), 1678-86.

46. Stauber M., Rapillard L., Van Lenthe G.H., Zysset P., Müller R. Importance of individual rods and plates in the assessment of bone quality and their contribution to bone stiffness. J Bone Miner Res. 2006;21(4), 586-95.

47. Pialat J.B., Vilayphiou N., Boutroy S., Gouttenoire P.J., Sornay-Rendu E., Chapurlat R., et al. Local topological analysis at the distal radius by HR-pQCT: Application to in vivo bone microarchitecture and fracture assessment in the OFELY study. Bone. Elsevier Inc.; 2012;51(3), 362-8.

48. Parfitt A.M. Implications of architecture for the pathogenesis and prevention of vertebral fracture. Bone. 1992;13(Suppl. 2), s41-7.

49. Liu X.S., Cohen A., Shane E., Stein E., Rogers H., Kokolus S.L., et al. Individual Trabeculae Segmentation (ITS)-based morphological analysis of high-resolution peripheral quantitative computed tomography images detects abnormal trabecular plate and rod microarchitecture in premenopausal women with idiopathic osteoporosis. J Bone Miner Res. 2010;25(7), 1496-505.

50. Walker M.D., Shi S., Russo J.J., Liu X.S., Zhou B., Zhang C., et al. A trabecular plate-like phenotype is overrepresented in Chinese-American versus Caucasian women. Osteoporos Int. 2014;25(12), 2787-95.

51. Liu X.S., Sajda P., Saha P.K., Wehrli F.W., Bevill G., Keaveny T.M., et al. 
Complete Volumetric Decomposition of Individual Trabecular Plates and Rods and Its Morphological Correlations With Anisotropic Elastic Moduli in Human Trabecular Bone. J Bone Miner Res. 2007;23(2), 223-35.

52. MacNeil J.A., Boyd S.K. Accuracy of high-resolution peripheral quantitative computed tomography for measurement of bone quality. Med Eng Phys. $2007 ; 29(10), 1096-105$

53. MacNeil J.A., Boyd S.K. Load distribution and the predictive power of morphological indices in the distal radius and tibia by high resolution peripheral quantitative computed tomography. Bone. 2007;41(1), 129-37.

54. Lai Y.M., Qin L., Hung V.W.Y., Chan K.M. Regional differences in cortical bone mineral density in the weight-bearing long bone shaft--a PQCT study. Bone. 2005;36(3), 465-71.

55. Wehner T., Claes L., Simon U. Internal loads in the human tibia during gait. Clin Biomech. Elsevier Ltd; 2009;24(3), 299-302.

56. Sasimontonkul S., Bay B.K., Pavol M.J. Bone contact forces on the distal tibia during the stance phase of running. J Biomech. 2007;40(15), 3503-9.

57. Perry J., k S.T., Davids J.R. Gait Analysis: Normal and Pathological Function. J Pediatr Orthop. 1992;12(6).

58. Chiba K., Burghardt A.J., Osaki M., Majumdar S. Heterogeneity of bone microstructure in the femoral head in patients with osteoporosis: An ex vivo HRpQCT study. Bone. Elsevier Inc.; 2013;56(1), 139-46. 
59. Liu X.S., Sajda P., Saha P.K., Wehrli F.W., Guo X.E. Quantification of the Roles of Trabecular Microarchitecture and Trabecular Type in Determining the Elastic Modulus of Human Trabecular Bone. J Bone Miner Res. 2006;21(10), 1608-17.

60. Samelson E.J., Broe K.E., Xu H., Yang L., Boyd S., Biver E., et al. Cortical and trabecular bone microarchitecture as an independent predictor of incident fracture risk in older women and men in the Bone Microarchitecture International Consortium (BoMIC): a prospective study. Lancet Diabetes Endocrinol. Elsevier; 2018;

61. Topliss C.J., Jackson M., Atkins R.M. Anatomy of pilon fractures of the distal tibia Patients and Methods. J Bone Jt Surg [Br]. 2005;87, 692-7.

62. Cohen J. Using Effect Size-or Why the P Value is Not Enough. J Grad Med Educ. 2012;(September), 279-82.

63. Geusens P., Chapurlat R., Schett G., Ghasem-Zadeh A., Seeman E., de Jong J., et al. High-resolution in vivo imaging of bone and joints: a window to microarchitecture. Nat Rev Rheumatol. Nature Publishing Group; 2014;10(5), 304-13. 


\section{Appendix: Result of linear correlation analyses}
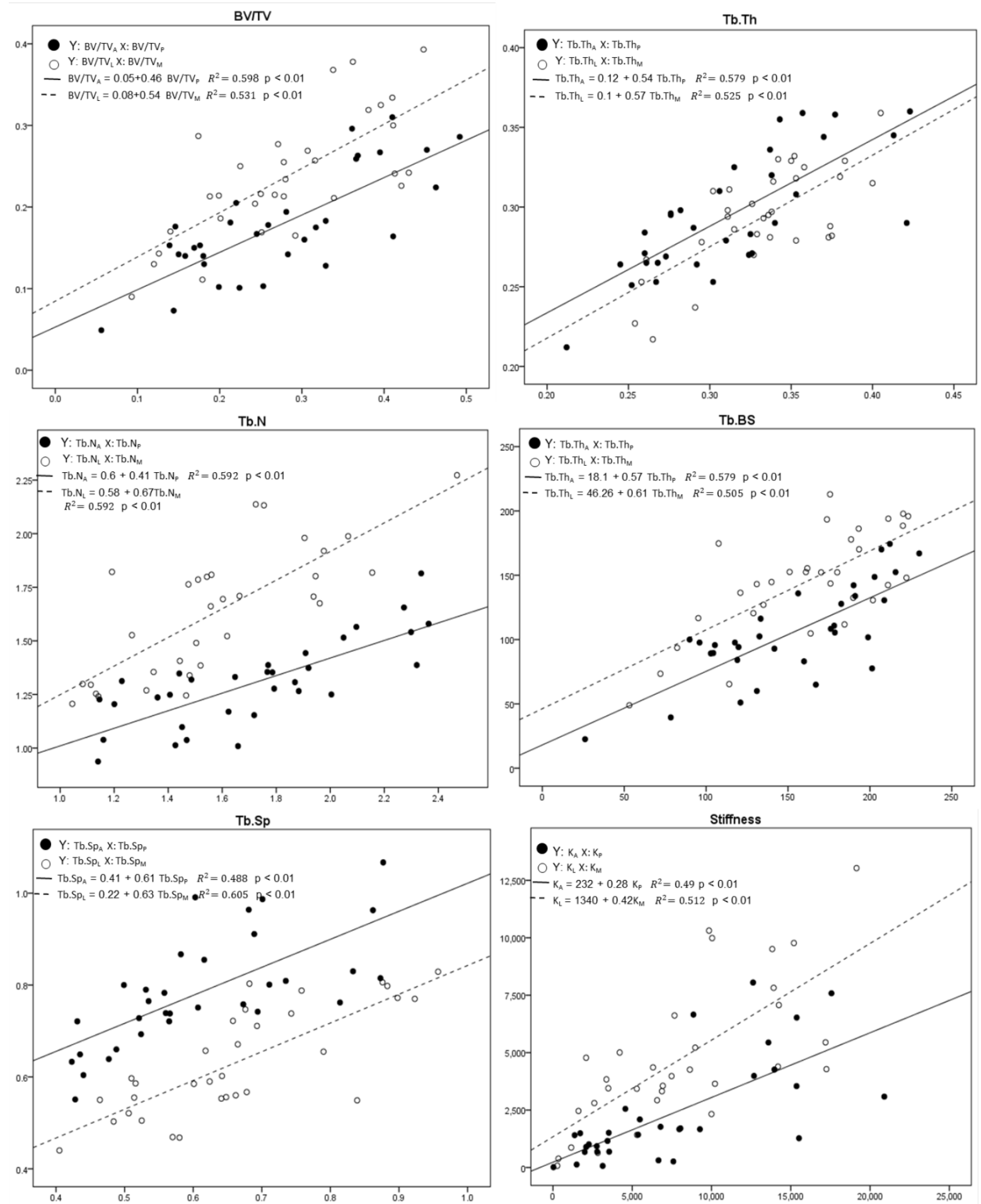

Figure A1: Results of linear correlation analyses between two anatomical regions: anterior and posterior (solid dots), lateral and medial (hollow dots) for various trabecular indices. 